Vol. 19(2):124-133, agosto - noviembre 2016

\title{
Tumores del sistema nervioso central en población pediátrica entre 0 y 14 años
}

\author{
Central nervous system tumors in pediatric population from \\ 0 to 14 years
}

\section{Tumores do sistema nervoso central em pacientes pediátricos entre 0 e 14}

\author{
Giselly Mayerly Nieves-Cuervo * \\ Edgar Fabián Manrique-Hernández * \\ Sergio Andrés Ojeda-Rincón * \\ Sneider Galvis-Pabón **
}

\section{Resumen}

Introducción: Los tumores pediátricos primarios del sistema nervioso central (SNC), ocupan el segundo lugar en frecuencia, superados solamente por las neoplasias hematológicas. Objetivo: Realizar una revisión y análisis de la literatura médica existente sobre tumores del SNC en población entre los 0 y 14 años centrado en la epidemiologia, factores etiológicos, manifestaciones clínicas y diagnóstico. Metodología: Se realizó una búsqueda en las bases de datos PubMed, MedlinePlus, BIREME y red interna de trabajos de grados y tesis de la Universidad Industrial de Santander. Además, se realizó revisión de las páginas de los entes gubernamentales encargados del registro epidemiológico sobre cáncer nacional e internacional. Resultados: Los tumores de SNC en la población pediátrica son la segunda causa de muerte infantil solo superado por la leucemia; tienen una clínica y factores etiológicos bien establecidos. La epidemiología no difiere en el mundo. Los síntomas más frecuentes son vómito, cefalea, ataxia, síntomas visuales y alteraciones motoras. Los factores etiológicos más representativos son virus, síndromes genéticos, infecciones maternas y perinatales, exposición a radiación electromagnética e ionizante; algunos son muy discutidos como la presencia de trauma al momento del nacimiento. Además, existen documentados factores protectores tales como consumo de antioxidantes, frutas y verduras, e historia reportada de alergias. Conclusiones: El adecuado entrenamiento a los médicos de atención primaria en la identificación de los signos y síntomas para la sospecha y diagnóstico de los estadios iniciales de estos tumores pueden disminuir los índices de mortalidad. [Nieves-Cuervos GM, Manrique-Hernández EF, Ojeda-Rincón SA, Galvis-Pabón S. Tumores del sistema nervioso central en población pediátrica entre 0 y 14 años. MedUNAB 2016; 19 (2): 124-133]

Palabras clave: Sistema Nervioso Central; Neoplasias del Sistema Nervioso Central; Pediatría; Neoplasias Encefálicas; Epidemiología; Signos y Síntomas.

\begin{abstract}
Introduction: The primary pediatric tumors of the central nervous system (CNS) rank second in frequency, only surpassed by hematological malignancies. Objective. Conduct a review and analysis of existing literature on CNS tumors in population between 0 and 14 focused on the epidemiology, etiological factors, clinical manifestations, and diagnosis. Methodology: The search for articles was conducted using the databases PubMed, MedLinePlus, BIREME and intranet degrees and thesis work of the Universidad Industrial de Santander. Further review of the pages from government agencies in charge of epidemiological
\end{abstract}

* Estudiante de Medicina de la Universidad Industrial de Santander, Bucaramanga, Colombia.

** Estudiante de Enfermería de la Universidad Industrial de Santander, Bucaramanga, Colombia.

Autor de correspondencia: Giselly Mayerly Nieves Cuervo. Correo electrónico: gisellymayi20@gmail.com.

Artículo recibido: 25 de marzo de 2015 Artículo aceptado: 10 de noviembre de 2016 
registry of cancer was made. Results: CNS tumors in pediatric population are the second leading cause of infant death second only to leukemia, have a clinic and etiological factors well established. The epidemiology don't differs in the world. The most common symptoms are vomiting, headache, ataxia, visual symptoms and motor disturbances. The most representative etiologic factors are viruses, genetic syndromes, maternal and perinatal infections, exposure to electromagnetic and ionizing radiation, some others are discussed as the presence of trauma at birth. There are also documented protective factors such as consumption of antioxidant, fruits and vegetables, and reported history of allergies. Conclusions: The adequate training of primary care physicians in the identification of signs and symptoms for suspicion and diagnosis of the initial stages of these tumors can reduce mortality rates. [Nieves-Cuervos GM, ManriqueHernández EF, Ojeda-Rincón SA, Galvis-Pabón S. Central nervous system tumors in pediatric population from 0 to 14 years. MedUNAB 2016; 19 (2): 124-133]

Key words: Central Nervous System; Central Nervous System Neoplasms; Pediatrics; Brain Neoplasms; Epidemiology; Signs and Symptoms.

\section{Resumo}

Introdução: Os tumores pediátricos primários do sistema nervoso central (CNS), classificação em segundo lugar na frequência, superado apenas pelo malignidades hematológicas. Objetivo: Revisar e análise da literatura

\section{Introducción}

Se define tumor de sistema nervioso como un crecimiento anormal de las células en los tejidos que conforman el cerebro que incluyen la masa encefálica, los nervios craneales, las meninges, las estructuras hematológicas y vasculares de los sistemas nerviosos central y periférico, además de los componentes neurales del sistema nervioso periférico. Estos tumores pueden ser catalogados como benignos (no cancerosos) o malignos (cancerosos) (1). Tienen gran importancia en indicadores de morbimortalidad, en especial en la población pediátrica, por lo que es una temática que debe ser abordada desde la teoría como la clínica, teniendo en cuenta los múltiples factores contribuyentes al desarrollo de enfermedades en esta población específica. En algunos casos la falta de información confiable y de alta calidad puede sesgar el abordaje antes mencionado.

Los tumores pediátricos primarios del sistema nervioso central (SNC), ocupan el segundo lugar en frecuencia, superados solamente por las neoplasias hematológicas, representando la principal causa de muerte por cáncer en la población pediátrica (2).

A su vez, los tumores embrionarios representan alrededor del $25 \%$ de los tumores primarios del SNC, entre ellos, el meduloblastoma (MB) y los tumores neuroectodérmicos primitivos (TNEP). En cuanto a incidencia, los tumores de existente sobre os tumores do SNC na população entre 0 e 14 anos, com foco em epidemiologia, fatores etiológicos, as características clínicas e diagnóstico. Metodologia: A pesquisa foi realizada no PubMed, MedlinePlus, NLM e graus de rede internos e tese de trabalho dos dados Universidade Industrial de Santander. Além disso, páginas de revisão de agências governamentais encarregadas de registro epidemiológico sobre câncer nacional e internacional foi realizada. Resultados: tumores do SNC na população pediátrica são a segunda principal causa de morte infantil perdendo apenas para a leucemia, têm fatores clínicos e etiológicos bem estabelecidos. Epidemiologia difere no mundo. Os sintomas mais comuns são vômitos, dor de cabeça, ataxia, sintomas visuais e distúrbios motores. Os fatores etiológicos mais representativos são os vírus, síndromes genéticas, infecções maternas e perinatais, a exposição às radiações electromagnéticas e ionizantes; alguns são muito discutidos como a presença de trauma no nascimento. Além disso, existe são documentados fatores de proteção, tais como o consumo de antioxidantes, frutas e legumes, e história de alergia relatados. Conclusões: $O$ treinamento adequado para médicos de cuidados primários na identificação dos sinais e sintomas de suspeita e diagnóstico dos estágios iniciais desses tumores pode diminuir as taxas de mortalidade. [Nieves-Cuervos GM, Manrique-Hernández EF, Ojeda-Rincón SA, Galvis-Pabón S. Tumores do sistema nervoso central em pacientes pediátricos entre 0 e 14. MedUNAB 2016; 19 (2): 124-133]

Palavras-chave: Sistema Nervoso Central; Neoplasias do Sistema Nervoso Central; Pediatria; Neoplasias Encefálicas; Epidemiologia; Sinais e Sintomas.

fosa posterior tienen la mayor representación; cerca del $40 \%$ de estos corresponde al MB, aunque algunos estudios posicionan el astrocitoma como el más común (3).

Los principales factores etiológicos reportados por la literatura son la radiación ionizante (4), la exposición a radiación electromagnética (5), el trauma al momento del nacimiento (6), y posiblemente algunos síndromes genéticos (7). Además, las características clínicas generales reportadas en los múltiples estudios son los signos focalizados (convulsiones, hemiparesias), alteraciones oculares y visuales, hipertensión intracraneana, alteración de los nervios craneales, endocrinopatías y el síndrome diencefálico(8).

El objetivo de este artículo es llevar a cabo una revisión y análisis de la literatura existente sobre los tumores del sistema nervioso central en la población entre 0 y 14 años, centrándose en la epidemiología, factores etiológicos, manifestaciones clínicas y diagnóstico para identificar los factores de riesgo y protectores, y las características clínicas para orientar a un diagnóstico más oportuno de la enfermedad.

\section{Metodología}

Se realizó una búsqueda de la literatura en bases de datos PubMed, MedlinePlus, BIREME y red interna de trabajos 
de grados y tesis de la Universidad Industrial de Santander (UIS). Además, se realizó efectuó revisión de las páginas de los entes gubernamentales encargados del registro epidemiológico sobre cáncer nacional e internacional: Sociedad Americana de Cáncer, Instituto Nacional de Cancerología ESE Colombia, Ministerio de Salud y Protección Social de Colombia, National Cancer Institute at the National Institutes of Health EEUU y Organización Mundial de la Salud (OMS). Los descriptores fueron: "Central Nervous System Tumors", "Central Nervous System Neoplasms", "Pediatrics" encontrados por medio de la base de datos MeSH. Encontrándose 150 artículos en la base de datos PubMed, dos en la base de datos MedlinePlus, 38 en la base de datos BIREME y 2 en la red interna de trabajos de grado de la UIS. Se seleccionaron 50 artículos. Como criterio de inclusión se tomaron los artículos que en revisión inicial tuvieran el abstract o resumen disponible, y en este se relacionara neoplasias de SNC y población pediátrica. No se utilizó ningún criterio de exclusión.

\section{Resultados}

\section{Epidemiología}

En la población pediátrica los tumores primarios del SNC ocupan el segundo lugar en frecuencia en la mayoría de centros oncológicos del mundo, superados solamente por las neoplasias hematológicas, representando la principal causa de muerte por cáncer en los niños (2).

Según la central de registro de tumores cerebrales de los Estados Unidos la tasa de incidencia de los tumores cerebrales primarios y del SNC malignos y no malignos en niños es de 5.1 casos por cada 100,000 niños. La incidencia es mayor en el sexo masculino con 5.2 frente a 5.1 casos en sexo femenino. Se estimó que para el año 2013 en los Estados Unidos habrá 4,300 casos nuevos en menores de 18 años, de estos se estima que 3,050 estén en menores de 15 años de edad $(9,10)$.

Según la edad, la incidencia de estos tumores varía. Entre el 2004 y el 2006 las tasas ajustadas por edad en menores de 1 año fueron de 40, entre 1 y 4 años de 47 , entre 5 y 9 años de 39 , entre 10 y 14 años de 36, entre 15 y 19 años de 42 por cada 100,000 habitantes (3). La tasa de incidencia de tumores del SNC reportada desde el año 2005 al 2009 en relación a la raza es mayor en blancos (3.4 casos), seguido por hispanos (2.5 casos), negros (2.3 casos), americanos asiáticos (2.1 casos) y nativos americanos de 1.7 casos por cada 100,000 habitantes (11).

Los tumores embrionarios representan el 25\% de los tumores primarios del SNC para la población pediátrica, siendo representados por el meduloblastoma (MB) y el Tumor Neuroectodérmicos primitivo (TNEP). Los tumores de fosa posterior son los de mayor incidencia, siendo cerca del $40 \%$ de estos el MB. Aunque algunos estudios posicionan el astrocitoma como el más común, seguido del $\mathrm{MB}(3)$.
En la población hispana, la tasa de incidencia de tumores cerebrales primarios y del SNC ajustada por edad para población menor de 14 años entre el año 2005 y 2009, fue de 2.7 casos por 100,000 habitantes. En cuanto a tumores embrionarios, la tasa de incidencia de los astrocitomas es $35 \%$ menor en niños hispanos que en los niños blancos no hispanos (12).

En Colombia, la distribución del cáncer en niños por grupos de edad según el Instituto Nacional de Cancerología, muestra que las neoplasias del SNC ocuparon en el año 2009 el segundo lugar, y el quinto lugar para el año 2010 y 2011 (10); sin embargo, a través de la historia, estos se han posicionado casi siempre entre el tercer y sexto lugar. Según el manual de Atención Integrada a las Enfermedades Prevalentes de la Infancia de Colombia, el porcentaje de presentación de cáncer del SNC en niños menores de 15 años representa el $22.1 \%$ de la totalidad de canceres en dichapoblación (12).

En Bucaramanga, en el año 2006 se realizó un estudio en el Hospital Ramón González Valencia, donde se encontró que la localización más frecuente de los tumores del SNC correspondieron a fosa posterior el $47.7 \%$, hemisferio derecho el $15.9 \%$, tallo cerebral el $13.6 \%$, de estos casos el reporte histopatológico más frecuente fue el MB, seguido de astrocitoma de bajo grado y el ependimoma. No se encontraron diferencias estadísticamente significativas según el sexo, edad del paciente y localización del tumor. Se encontró que la edad de mayor presentación de tumores cerebrales es entre los 5 y 9 años de edad(13).

\section{Factores etiológicos}

La radiación ionizante: se ha relacionado con la aparición de tumores cerebrales, por ejemplo, en aquellos pacientes que recibieron tratamiento de otro tipo de cáncer (leucemia linfocítica aguda) $(4,14)$. La exposición infantil a la toma de tomografía axial computarizada de cráneo también puede estar asociada con un mayor riesgo de tumores cerebrales. Algunos autores han referenciado que el tumor más comúnmente asociado a la radiación son los gliomas malignos seguidos por los meningiomas y los astrocitomas de bajo grado (15-17).

La exposición radiación electromagnética: aumenta el riesgo 2.4 veces (18), aunque no está asociado a la exposición de fuentes de calor eléctricas o aparatos electrónicos de uso cotidiano (5).

Presencia de trauma al momento del nacimiento: por cualquier mecanismo, entre los cuales se han descrito parto en madre primeriza, uso de fórceps o trabajo de parto prolongado, aunque no es decisivo si aumenta o no el riesgo de presencia de cáncer cerebral, algunos estudios lo confirman y algunos lo niegan (19).

Infecciones maternas y perinatales: aunque la asociación aun no es clara, algunos estudios han encontrado mayor riesgo para todos los tipos de tumores cerebrales (6). 
Síndromes genéticos: la literatura describe algunos síndromes que aumentan el riesgo de desarrollar tumores del SNC (7), entre los cuales se encuentra la neurfibromatosis (20), síndrome de von Hippel-Lindau, síndrome de Li Fraumeni, y síndrome de Turcot (21). En cuanto a mutaciones específicas se ha asociado al tumor teratoideo/rabdoide atípico el gen SMARCB1, y alteraciones genómicas del BRAF, en especial la fusión del KIAA1549 y BRAF (22), y la activación de la vía ERK/MAPK; también se ha observado asociación a mutaciones en histona H3.3 y mutaciones en el gen TP5325, aunque en menor frecuencia (23).

Malformaciones congénitas de SNC no asociadas a defectos cromosómicos: de estas se ha documentado relación con un aumento de riesgo en el cáncer de SNC $(\mathrm{HR}=2.40,95 \% \mathrm{CI}: 1.43-4.02)(24)$.

Virus: un estudio realizado por Walshet et al., argumenta que el parvovirus (SV-40) puede ser un factor etiológico importante, debido a un aumento estadísticamente significativo en la incidencia de este tipo de tumores (específicamente MB, neuroblastoma, retinoblastoma, astrocitoma) en niños inmunizados con la vacuna del polio salk contaminada con el virus anteriormente mencionado (24).

Antioxidantes, frutas y verduras: su consumo parece reducir el riesgo de tumores cerebrales, y también se ha documentado una disminución de la incidencia de glioma en niños con una historia reportada de alergias $(25,26)$.

\section{Clasificación de los Tumores del SNC}

Los tumores de SNC actualmente se clasifican según la cuarta edición de la Organización Mundial de la Salud (OMS), la cual muestra una adecuada correlación clínico patológica y un enfoque en el pronóstico. Los tumores del SNC presentan una gran variedad de apariencias histológicas y derivaciones citológicas, debido a la complejidad del órgano al cual están relacionados. Esta clasificación se basa en la morfología, localización, distribución de edad y comportamiento biológico. Además, incluye criterios como el estado neurológico, las alteraciones genéticas, los hallazgos radiológicos, la extensión de la resección quirúrgica, si esta es posible y los índices de proliferación $(27,28)$.

La OMS en un sentido global caracteriza los tumores del SNC según el sistema u órgano comprometido: tumores derivados del neuroepitelio, tumores de nervios craneales y paraespinales, tumores de las meninges, tumores de células germinales, linfomas y neoplasias hematopoyéticas, tumores metástasicos y tumores de la región selar $(27,28)$.

Dentro de los tumores derivados del neuroepitelio encontramos grupos como son: tumores astrocitícos, tumores oligodendrogliales, tumores oligodendroastrocíticos, tumores ependimales, tumores de plexos coroideos y tumores de la región pineal. Cabe destacar que los tumores derivados del neuroepitelio son los que han supuesto mayor dificultad a la hora de definir patología tumoral con base común genética e histológica, debido a su gran variedad morfológica y citológica, así como por su posibilidad de evolucionar hacia formas malignas $(29,30)$.

\section{Gradación de los tumores del SNC}

El sistema de gradación de los tumores del SNC surgió como complementariedad al sistema de clasificación, con el fin de contribuir en el estudio pronóstico de esta neoplasia puesto que utiliza algunos datos clínicos y genéticos, además de variantes histológicas, convirtiendo la gradación en un factor importante para predecir el comportamiento biológico del tumor, y por lo tanto para la toma de decisiones terapéuticas. Este sistema es una escala de malignidad basada en criterios estrictamente histológicos previstos en las anteriores clasificaciones de la OMS; sin embargo, incluye algunas nuevas entidades tumorales, tales como el glioma angiocéntrico, tumor papilar de la región pineal, entre otros (30).

El sistema de gradación histológica se estratifica por grados, de I al IV, siendo I y II de grado bajo y III y IV de alto grado, definiéndose cada grado de la siguiente manera:

[ $]$ Grado I: Incluye lesiones con bajo potencial proliferativo, crecimiento expansivo, posibilidad de cura luego de la resección quirúrgica.

$\square$ Grado II: Lesiones con bajo potencial proliferativo, crecimiento infiltrante y tendencia a la recurrencia.

$\square$ Grado III: Lesiones con evidencia histológica de malignidad, mitosis, anaplasia y capacidad infiltrativa.

$\square$ Grado IV: Lesiones malignas, mitóticamente activas, tendencia a la necrosis y evolución rápida tanto pre como postoperatoria.

Este sistema de gradación es apenas un componente más de una combinación de criterios que permiten predecir la respuesta a una futura acción terapéutica. Para cada entidad tumoral es necesario tener en cuenta otros criterios tales como la localización, la edad, los hallazgos clínicos y radiológicos, los índices de proliferación, que en conjunto permiten predecir estimar un comportamiento pronóstico. A pesar de incluir esta gran combinación de criterios, el pronóstico en pacientes con grado II tienen una supervivencia de 5 años aproximadamente, los grado III de 2-3 años y los grado IV depende del régimen terapéutico aplicado (31).

\section{Entidades tumorales especiales y sus características}

Para la población pediátrica es necesario centrarse en los tumores embrionarios, y orientar una pequeña sección hacia los pineoblastomas. En cuanto a los tumores embrionarios, corresponden al $20 \%$ de todos los tumores de SNC en población pediátrica, su clasificación incluye el meduloblastoma, el TNEP del SNC (Neuroblastoma del $\mathrm{SNC}$, ganglioneuroblastoma del SNC, meduloepitelioma, ependimoblastoma) y tumor teratoideo/rabdoide atípico (3). 
Si se habla de meduloblastoma, por definición estas neoplasias pueden ser de fosa posterior y se dividen en 5 tipos histológicos: clásico, anaplásico, de células grandes, nodular desmoplásico y con modularidad extensa o MBAN. La variante desmoplásica es más común en lactantes y adolescentes, el subtipo nodular es de predominio también en lactantes y es de buen pronóstico, los meduloblastomas al tener una gran carga genética en los niños tiene gran valor predictivo conocer su subtipo biológico y molecular $(31,32)$.

Los pineoblastomas son tumores del parénquima pineal, que en cuanto a tratamiento y pronóstico son muy parecidos a los tumores embrionarios. Según su tipo celular pueden ser astrocitomas, tumores oligodendrogliales, gliomas mixtos y tumores neuronales. Según la OMS se determinó que la variante pilomixoide del astrocitoma pilocítico pasa de ser tumor grado I a convertirse en grado II. Aunque pueden presentarse en cualquier lugar del SNC, cada variante tiene predilección por ciertos lugares; por ejemplo, el astrocitoma pilocítico ( $80 \%$ de los astrocitomas) se localiza preferentemente en el nervio óptico, quiasma óptico e hipotálamo, tálamo y ganglios basales, hemisferios cerebrales, cerebelo, tronco encefálico y, raramente, médula espinal (33).
En cuanto a los tumores de células germinativas del sistema nervioso central se ha descrito mayor incidencia en sexo masculino, se describe que tienen como región de aparición la pineal (dos veces más frecuentes) o supraselar, teniendo como característica lesiones solitarias o múltiples (34). También se han documentado como áreas de aparición, aunque poco comunes, los núcleos basales, los ventrículos, el tálamo, los hemisferios cerebrales y la médula (35).

En la tabla 1 se muestra la clasificación de los tumores del SNC según su localización según la OMS (30).

\section{Características clínicas de los tumores del SNC}

La clínica de este tipo de tumores en población pediátrica cambia con la ubicación del tumor y la edad del niño en el momento de la presentación (30). Los síntomas y signos suelen ser de difícil identificación e inespecíficos (36).

Se ha reportado en general, hasta un $38.7 \%$ de los pacientes presentan como síntoma inicial una crisis convulsiva y más del $50 \%$ sufrirá alguna a lo largo de su enfermedad (37). Seguido de cefalea, en un $35.2 \%$ de los casos, siendo este el síntoma más frecuente al momento del diagnóstico,

Tabla 1. Clasificación de los Tumores Cerebrales según la Organización Mundial de la Salud, 2007

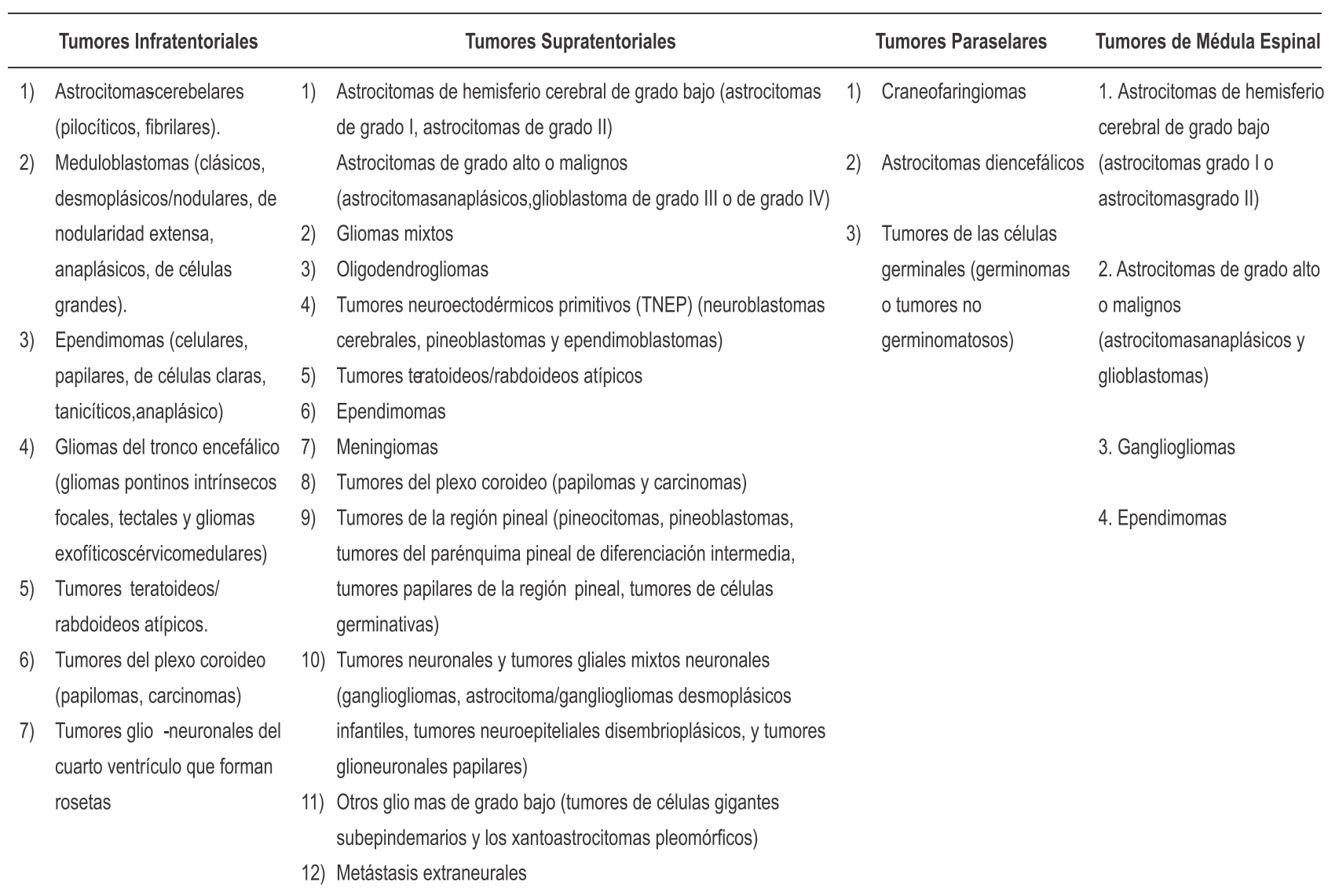

Fuente: Adaptado de Louis DN, the 2007 WHO classification of tumours of the central nervous system (30) 
probablemente debido al aumento de la presión intracraneana $(38,39)$.

En orden de frecuencia le siguen alteración en la marcha, papiledema, estrabismo, cambios de comportamiento, macrocefalia, parálisis de los nervios craneales, letargia, movimientos anormales de los ojos, hemiplejia, pérdida de peso y alteración de la conciencia $(8,40)$.

La historia natural de enfermedad puede presentar $(8,13)$ :

미 Signos localizados: convulsiones, hemiparesias, perdida hemisensorial e hiperreflexia.

ㄸAteraciones oculares y visuales: amaurosis unilateral, ausencia reflejo pupilar, ausencia de reflejo consensual, alteraciones campos visuales.

미ipertensión intracraneana: cefalea, vomito, ataxia troncal, dismetría, nistagmos, incoordinación de extremidades.

पIIAlteración de los nervios craneales: parálisis de V, VI, VII, IX par, diplopía, alteración del habla, alteración para deglutir, incapacidad para conjugar la mirada, incapacidad para lateralizar la mirada.

口[पEndocrinopatías: diabetes insípida, alteraciones de crecimiento, disminución de la visión, alteración de los campos visuales.

口WSíndrome diencefálico: falla del crecimiento pondoestatural, emaciación, euforia, buen apetito.

\section{Signos y síntomas según el tipo de cáncer}

Astrocitoma infantil: su sintomatología puede variar dependiendo del origen del tumor (cerebro o la médula espinal), tamaño del tumor, velocidad de crecimiento y la edad de aparición. Los síntomas que se presentan son dolor de cabeza al despertar o que desaparece después de vomitar, náusea, emesis, cambios visuales, auditivos o en la capacidad de habla, pérdida de equilibrio y problemas para caminar, debilidad o cambio en la sensibilidad de un lado del cuerpo, somnolencia, cambios de comportamiento, crisis convulsivas, pérdida o aumento de peso sin razón conocida y en los lactantes aumento del tamaño de la cabeza (41).

Astrocitomacerebeloso: representan el 12\% de los tumores intracreaneanos en los niños, las manifestaciones más frecuentes son cefalea y posteriormente la sintomatología asociada al síndrome de hipertensión endocraneana, en los lactantes cuyas fontanelas no se han cerrado aún, presentan síntomas tales como irritabilidad, vómito y aumento del perímetro cefálico debido a la obstrucción y su consiguiente hidrocefalia. En su evolución clínica puede presentarse hipotonía, dismetría, adiadococinesia, lateralización de la marcha al mismo lado del tumor, ataxia del tronco y voz escandida (42).

Meduloblastomas: debido a que en su mayoría surgen en la región del cuarto ventrículo su sintomatología temprana se relaciona con la obstrucción del líquido cefalorraquídeo (LCR) y la hidrocefalia resultante, por lo que se puede encontrar aparición abrupta de dolores de cabeza, vómitos, letargo, inestabilidad, nistagmo y/o papiledema. En los lactantes, la presentación varía, incluyendo letargo inespecífico, retrasos psicomotores, pérdida de los hitos del desarrollo y dificultades en la alimentación $(7,20)$.

Pineoblastomas: se presentan en los últimos años de la niñez, representan aproximadamente el $4 \%$ de los tumores intracraneales. Su clínica se caracteriza por hidrocefalia, debido a la obstrucción del LCR a la altura del tercer ventrículo, anomalías en los movimientos oculares, manifestados en pérdida de la mirada hacia arriba, nistagmo de convergencia, y retracción palpebral $(7,20$, $30)$.

Glioblastoma multiforme: principalmente afecta el lóbulo frontal, entre sus síntomas más frecuentes se encuentran cefalea, náuseas o vómitos y convulsiones. El papiledema es el hallazgo físico más común, también se puede presentar ataxia, parálisis de los nervios craneales y paresias (43).

Glioma del tronco encefálico infantil y tumor teratoideo/ rabdoide atípico: sus hallazgos clínicos más frecuentes son pérdida del equilibrio, problemas visuales y auditivos, cefalea matinal o que desaparece después de vomitar, náuseas y emesis, somnolencia inusual (8).

Pieloblastoma: hidrocefalia por obstrucción del LCR en el tercer ventrículo con su consiguiente sintomatología y otros síntomas por presión en la parte posterior del tronco cerebral, como anomalías en los movimientos oculares (pupilas con poca reactividad a la luz, pero con reflejo de acomodación conservado, pérdida de la mirada hacia arriba, retracción o nistagmo de convergencia, y retracción palpebral (44-46).

Tumores de células germinativas del sistema nervioso central infantil: polidipsia, poliuria, pubertad precoz, disminución en la visión, baja estatura, cefalea, náuseas, vómito, astenia, adinamia, disminución en el rendimiento escolar (44).

Lesiones supratentoriales: suelen dar lugar a deficiencias neurológicas focales, tales como hemiparesia, convulsiones y pérdida del campo visual, esto depende de qué parte de la corteza cerebral se encuentre afectada (47-50).

\section{Diagnóstico de los Tumores del SNC}

La historia clínica y la exploración son la base del diagnóstico, pues estas permiten la sospecha y búsqueda rápida de esta lesión, pero el diagnóstico definitivo se basa en la identificación de la lesión por neuroimagen, ya sea por resonancia magnética o por tomografía axial computarizada. Posteriormente, se requiere un examen histológico para hacer un diagnóstico del tipo de tumor específico (51).

Los tumores cerebrales infantiles dependen de la localización del tumor, la biología del tumor y la edad del niño, dificultando la identificación de los síntomas, lo que se asocia con una mayor morbilidad, aumentando la 
probabilidad de deterioro cognitivo y trastornos psicosociales $(45,52)$.

Existen diversos marcadores tumorales en sangre o LCR, que son importantes para la orientación del tipo de tumor, además, son útiles para la valoración del grado de resección, la respuesta al tratamiento y la presencia de recidiva, pero, como se escribió con anterioridad, el diagnóstico definitivo se hace por medio de neuroimagen, ubicándose en primer lugar la resonancia magnética seguida de la tomografía axial computarizada, siendo las técnicas más destacadas las siguientes $(29,53)$ :

Tomografía axial computarizada (TAC): la TAC simple y contrastada sigue siendo una alternativa válida en ocasiones donde no se cuenta con la posibilidad de realizar una resonancia magnética (53). La TAC proporciona datos sobre: a) presencia o ausencia de tumor; b) tamaño del mismo; c) forma y densidad tumoral; d) localización; e) comportamiento tras la administración de contraste; f) calcificaciones, zonas de necrosis y quistes; g) edema peritumoral; h) desplazamientos y herniaciones cerebrales; i) afectación de estructuras óseas; j) presencia de hidrocefalia; k) hemorragia tumoral y otros (29).

Resonancia magnética (RM): la RM proporciona $(46,53)$ : 1. La posibilidad de efectuar cortes en cualquier plano y dirección del espacio: axial, sagital, coronal y oblicuo.

2. Alta resolución de contraste.

3. Puede detectar rotura de la barrera hematoencefálica mediante el uso de contraste intravenoso paramagnético (gadolinio).

4. La RM ofrece un buen mapa vascular no invasivo, en ocasiones sin necesidad de administrar contraste intravenoso.

Angiografía cerebral: constituye el estudio de elección en sospecha de lesiones vasculares (aneurisma y angiomas). La realización de una angiografía cerebral está indicada en tumores con gran vascularización para conocer los vasos afluentes a la neoplasia, sobre todo si se contempla la posibilidad de embolización preoperatoria como parte del tratamiento (45).

Tratamiento de los tumores del SNC

Las principales opciones de tratamiento se enmarcan en cirugía, quimioterapia y radioterapia. Hay que destacar aquí que el tratamiento de los tumores del SNC en los niños requiere un abordaje en equipo, coordinando las diversas especialidades que colaboran en el tratamiento en Comités de Neuro-Oncologia Pediátrica. Por otra parte, se hace necesaria la participación de estas unidades en estudios cooperativos nacional e incluso supranacionales para poder adquirir la suficiente experiencia que requiere la práctica de la medicina basada en la evidencia.

Algunos tumores poseen una sensibilidad notablemente alta a los tratamientos de radioterapia y quimioterapia, una vez determinado el diagnóstico histológico sin la necesidad de una resección quirúrgica. De la misma manera en tumores en áreas elocuentes la resección quirúrgica puede no ser posible (51). Para la mayoría de los tumores la opción preferida es el abordaje directo, con intención de excéresis total, o lo más amplia posible, dependiendo de la naturaleza del tumor y de su localización, siendo lo anterior esencial para la posterior eficacia de la radioterapia y quimioterapia (51).

Cirugía: cumple dos objetivos principales que son establecer un diagnóstico de certeza y reducir el volumen tumoral. La opción preferida es el abordaje directo con intención de exeresis total o lo más amplia posible, para ello existen técnicas como: la microcirugia, técnicas de cirugía mínimamente invasiva, localización intraoperatoria tumoral mediante ultrasonidos, registros neurofisiológicos intraoperatorios, neuro-navegación, láser, aspirador ultrasónico, TAC intraoperatoria (45).

Radioterapia: entre los factores a considerar para el inicio de radioterapia se encuentra el tiempo adecuado de aplicación, la modalidad más apropiada, la toxicidad a corto y largo plazo para la radiación sola y en combinación con quimioterapia. Se deben tener en cuenta los efectos de la radiación ionizante en el sistema nervioso, debido a que muchos pacientes recibirán radioterapia en algún momento de su tratamiento, previo consentimiento informado de los padres o tutores (51). Por otro lado, la radioterapia local está indicada en tumores malignos, en propensos a recidivar, en exéresis incompletas, e incluso en tumores de bajo grado inextirpables $(45,47)$.

Quimioterapia: se emplea en tumores como el meduloblastoma de alto riesgo y los astrocitomas de alto grado; la quimioterapia puede seguir a la radioterapia, o puede precederla, puesto que reducen los niveles de radiación necesarios y ayuda al control del tumor si se aplica antes (48). Las ventajas que se aducen para la quimioterapia postoperatoria son: i) el aprovechamiento de la ruptura de la barrera hematoencefálica, producida por la cirugía; ii) la quimioterapia es más eficaz antes de que la radioterapia produzca cambios vasculares que impiden el paso de estas drogas (45).

\section{Diagnóstico diferencial}

Teniendo en cuenta que los signos y síntomas de los tumores del SNC en niños son inespecíficos, estos pueden ser mal diagnosticados como enfermedades comunes de la infancia, como la gastroenteritis viral, y cefalea tensional y migraña (46).

La cefalea se manifiesta a lo largo de horas o días, por lo que se clasifica como subaguda, por lo que es necesario para el clínico tener en cuenta los signos característicos de cada una de sus causas para un correcto diagnóstico diferencial. En caso de tumores de SNC la cefalea puede ser causada por hipertensión intracraneal debido al tumor, acompañada de los otros síntomas y signos secundarios; o por hidrocefalia, 
un absceso cerebral, entre otros. Por lo tanto, debe ser confirmada su etiología por medio de TAC o RM cerebral (49).

El vértigo, principalmente a causa de tumores de fosa posterior, característicamente es leve, dura meses y está acompañado de ligero nistagmo, puede haber presencia de papiledema bilateral, abolición del reflejo corneal ipsilateral o signos de lesión de los pares craneales V, VI, VII, X, y XI, además de signos cerebelosos ipsilaterales y signos piramidales contralaterales, estas características son esenciales para sus sospecha y así realizar una resonancia magnética para el descubrimiento del tumor. Si esto no se cumple podría confundirse con una epilepsia del lóbulo temporal, insuficiencia vertebrobasilar, vértigo posicional benigno o alguna patología del oído medio (50).

Los diagnósticos diferenciales proporcionados por neuroimagen son de tipo: a) infecciosas: tuberculosis, absceso piógeno, cisticercosis, toxoplasmosis, micosis, virales, sarcoidosis. b) Vasculares: aneurismas, malformaciones arterio-venosas, vasculitis primaria del sistema nervioso, hemangiomas, cavernomas, evento vascular cerebral. c) Otras: radionecrosis, cerebritis, lesiones quísticas benignas, disgenesias cerebrales focales (51).

\section{Conclusiones}

Los tumores del SNC son una entidad que afecta a un gran porcentaje de la población pediátrica, siendo la segunda causa de muerte infantil, solo superado por la leucemia, que, si bien no tiene una forma identificada de prevención en la actualidad, con una sospecha temprana y un diagnóstico eficiente puede disminuir los índices de morbimortalidad. Por lo tanto, es importante entrenar al médico de atención primaria en la correcta identificación de los signos y síntomas cardinales para la identificación eficaz y así obtener una remisión en estadios tempranos del tumor, pues, aunque estos son inespecíficos, debe estar dentro de los posibles diagnósticos diferenciales una patología de este tipo.

Los síntomas más frecuentes son vómito, cefalea, ataxia, síntomas visuales y alteraciones motoras. Es evidente la falta de información concluyente en cuanto a factores de riesgo o etiológicos; sin embargo, los más representativos son virus, síndromes genéticos, infecciones maternas y perinatales, exposición a radiación electromagnética e ionizante; y algunos otros discutidos como la presencia de trauma al momento del nacimiento. Además, existen documentados factores protectores tales como consumo de antioxidantes, frutas y verduras, e historia reportada de alergias.

La epidemiología de Colombia es escasa, encontrándose solo 2 estudios nacionales de esta población, por lo que se abre un campo para investigaciones futuras.

\section{Referencias}

1. Instituto nacional del cáncer de los institutos de salud de Ios EEUU disponible en:http://www.cancer.gov/ espanol/tipos/cerebro. Fecha de consulta: 26 junio 2014

2. Homer MJ, Ries, LAG, Krapcho, M, et al (eds). SEER Cancer Statistic Review, 1975-2006 http:// seer.cancer.gov/csr/1975_2006/

3. Smoll NR, Drummoñd KJ.The incidence of medulloblastomas and primitive neurectodermal tumours in adults and children. J Clin Neurosci. 2012, 19 (11): 1541-1544.

4. Braganza MZ, Kitahara CM, Berrington de González A, Inskip PD, Johnson KJ, Rajaraman P. Ionizing radiation and the risk of brain and central nervous system tumors: a systematic review. Neuro Oncol. 2012; 14: 1316-1324.

5. Sorahan T, Hamilton L, Gardiner K, Hodgson JT, Harrington JM. Maternal occupational exposure to electromagnetic fields before, during, and after pregnancy in relation to risks of childhood cancers: findings from the Oxford Survey of Childhood Cancers, 1953-1981 deaths. Am J Ind Med. 1999; 35(4): 348-357

6. Linet MS, Gridley G, Cnattingius S, Nicholson HS, Martinsson U, Glimelius B, et al. Maternal and perinatal risk factors for childhood brain tumors (Sweden). Cancer Causes Control. 1996; 7(4): 437-438.

7. Weiss W. Genetics of Brain Tumors. Rev Current opinion in pediatrics $2000 ; 12: 543-48$.

8. National Cancer Institute at the National Institutes of Health.Tumorteratoideo/rabdoideatípicodelsistemanerv ioso central infantil. Tomado de: http://www.cancer.gov/ espanol/pdq/tratamiento/ATRT-SNC-infantil/Health Professional. Fecha de consulta: 02 de marzo del 2013.

9. Cbtrus.org [Internet]. Estados Unidos de América: Central Brain Tumor Registry Of The United States; 2012 [citado 23 de junio de 2014] Disponible en: http://www.cbtrus.org/factsheet/factsheet.html

10. Instituto Nacional de Cancerología ESE Colombia. Cáncer en cifras, registro institucional de cáncer. Tomado de: http://www.cancer.gov.co/documentos/ Tablas2011/Tabla\%204.pdf. Fecha de consulta: 20 de febrero del 2014.

11. Sociedad Americana del Cáncer. Datos y Estadísticas sobre el Cáncer entre los Hispanos/Latinos 20122014.American Cancer Society. 2012: 1-40.

12. Ministerio de Salud y Protección Social, Organización Panamericana de la Salud. Atención Integrada a las Enfermedades Prevalentes de la Infancia. 3ra edición. Colombia: Organización Panamericana de la Salud; 2012.

13. Sierra Rosales G. 2006. Tumores del sistema nervioso central. Presentación durante un periodo de 5 años en el Hospital Universitario Ramón González Valencia de Bucaramanga. Tesis de grado. Bucaramanga, Universidad Industrial de Santander: 1-71.

14. Relling MV, Rubnitz JE, Rivera GK, Boyett JM, Hancock $M L$, Felix CA, et al. High incidence of secondary brain tumours after radiotherapy and antimetabolites. Lancet. 1999; 354:34-39.

15. Pearce MS, Salotti JA, Little MP, McHugh K, LeeC, Kim $\mathrm{KP}$, et al. Radiation exposure from CT scans in childhood and subsequent risk of leukaemia and brain tumours: a retrospective cohort study. Lancet. 2012; 380: 499-505. 
16. Davis F, Il'yasova D, Rankin K, McCarthy B, Bigner DD. Medical diagnostic radiation exposures and risk of gliomas. Radiat Res. 2011; 175: 790-796.

17. Pettorini BL, Park YS, Caldarelli M , Massimi L , Tamburini G , Di Rocco C. Radiation-induced brain tumors after central system irradioation in childhood : a review. Chlids Nervs Syst 2008; 24(7): 793-805.

18. Wertheimer N, Leeper E. Electrical wiring configurations and childhood cancer. Am J Epidemiol 1979; 109: 273284.

19. Choi NW, Schuman LM, Gullen WH. Epidemiology of primary central nervous system neoplasms. II. Casecontrol study. Am J Epidemiol 1970; 91: 467-485.

20. Goutagny S, Kalamarides M. Meningiomas and neurofibromatosis. J Neurooncol 2010; 99: 341-347.

21. Martinez M, García Ribes A, GaraizarAxpe C. Tumores cerebrales infantiles: diagnóstico y semiología neurológica. Protocolos diagnósticos terapéuticos de la AEP: Neurología pediátrica. 2008.

22. $P$ fister $S$, Janzarik WG, Remke M, Ernst A, Werft W, Becker $\mathrm{N}$, et al.: BRAF gene duplication constitutes a mechanism of MAPK pathway activation in low-grade astrocytomas. J Clin Invest. 2008; 118(5): 1739-1749.

23. Jones DT, Kocialkowski S, Liu L, PearsonDM, Ichimura $\mathrm{K}$, Collins VP. Oncogenic RAF1 rearrangement and a novel BRAF mutation as alternatives to KIAA1549:BRAF fusion in activating the MAPK pathway in pilocytic astrocytoma. Oncogene. 2009; 28(20): 2119-2123.

24. Sun Y, Overvad K, Olsen J. Cancer risk in children with congenital malformations in the nervous and circulatory system-A population chort study. Cancer Epdemiol. 2014; 38(4): 393-400

25. Walsh JW, Zimmier SG, Perdue MI. Role of viruses in the induction of primary intracranial tumors. Neurosurgery. 1982; 10: 643-662

26. Michaud DS, Holick CN, Batchelor TT, Giovannucci E, Hunter DJ. Prospective study of meat intake and dietary nitrates, nitrites, and nitrosamines and risk of adult glioma. Am J Clin Nutr. 2009; 90: 570-577.

27. Louis DN, Ohgaki H, Wiestler OD, Cevencee WK, Burger PC, Jouvet A, et al. The 2007 WHO classification of tumours of the central nervous system. Acta Neuropathol. 2007; 114(2): 97-109.

28. Smith MA, Seibel NL, Altekruse SF, Ries LA, Melbert DI, O'Leary M, Smith Fo, et al. Outcomes for children and adolescents with cancer: challenges for the twenty-first century. J Clin Oncol. 2010; 28(15): 2625-2634.

29. Villarejo F, Martinez JF. Tumores cerebrales en niños. Lage Pediatr Integral 2012; XVI (6): 475-486

30. Packer RJ, Rorke-Adams LB, Lau CC, et al. Embryonal and pineal region tumors. In: Pizzo PA, Poplack DG, eds.: Principles and Practice of Pediatric Oncology. 6th ed. Philadelphia, Pa: Lippincott Williams and Wilkins, 2011:772-808

31. Garrè ML, Cama A, Bagnasco F, Morana G, Giangaspero F, brisigotti M, et al. Medulloblastoma variants: age-dependent occurrence and relation to Gorlin syndrome--a new clinical perspective. Clin Cancer Res. 2009; 15(7): 2463-2471.

32. Northcott PA, Jones DT, Kool M, Robinson GW, Gilbertson RJ, Cho YJ, et al. Medulloblastomics: the end of the beginning. Nat Rev Cancer. 2012; 12(12): 818-834.
33. National Cancer Institute at the National Institutes of Health. Astrocitomas infantiles. Tomado de: http://www.cancer.gov/espanol/pdq/tratamiento/astrocit omas-infantiles/HealthProfessional. Fecha: 02 de marzo del 2013

34. Weksberg DC, Shibamoto Y, Paulino AC. Bifocal intracranial germinoma: a retrospective analysis of treatment outcomes in 20 patients and review of the literature. Int J RadiatOncolBiolPhys. 201282 (4): 13411351

35. Goodwin TL, Sainani K, Fisher PG: Incidence patterns of central nervous system germ cell tumors: a SEER Study. J PediatrHematolOncol. 2009; 31(8): 541-4.

36. Wilne SH, Dineen RA, Dommett RM, Chu TP, Walker DA. Identifying brain tumours in children and young adults. BMJ. 2013; 347: f5844

37. Preston-Martin S. Epidemiology of primary CNS neoplasms.NeurolClin 1996;14:273-290.

38. Guillen Porta V. Tumores cerebrales primarios y metastásicos. Servicio de oncología médica fundación instituto calenciano de oncología; 2008: 21-24.

39. Sophie Wilne, Jacqueline Collier, Colin Kennedy, Karin Koller, Richard Grundy, David Walker Presentation of childhood CNS tumours: a systematic review and metaanalysis. The Lancet Oncology (Impact Factor: 25.12). 09/2007; 8(8):685-95.

40. Wilne S, Collier J, Kennedy C, Koller K, Grundy R, Walker D. Presentation of childhood CNS tumours: a systematic review and meta-analysis. LancetOncol. 2007;8(8):685.

41. National Cancer Institute at the National Institutes of Health. Información general sobre los astrocitomas infantiles. Tomado de: http://www.cancer.gov/espanol/ pdq/tratamiento/astrocitomas-infantiles/patient. Fecha: 01 de marzo del 2013.

42. Diaz R. 2014. Tumores cerebrales en la infancia. En: Fundamentos de Pediatría (Correa JA). corporacion para investigaciones biológicas. Colombia 2015:110.

43. Dohrmann GJ, Farwell JR, Flannery JT. Glioblastomamultiforme in children.Journal of Neurosurgery 1976; 44(4):442-8.

44. National Cancer Institute at the National Institutes of Health.Tumores de células germinativas del sistema nervioso central infantil. Tomado de: http:// www.cancer.gov/espanol/pdq/tratamiento/celulasgerm inales-SNCinfantil/patient. Fecha: 02 de Marzo del 2013.

45. Wilne S, Koller K, Collier J, et al. Thediagnosis of brain tumours in children: a guideline to assist healthcare professionals in the assessment of children who may have a brain tumour. ArchDisChild. 2010; 95:534

46. Steinbok P, Heirschel S, Cochrane DD, Kestle JR. Valve of postoperative surveillance imaging in the management of children with some common brain tumors. J Neurosurg. 1996; 84: 726-732.

47. Thorp NJ, Taylor RE. management of central nervous system tumors in children. ClinOncol(R CollRadiol) 2014; 26(7):438-445.

48. Dufour C, Guerrini-Rousseau L, Grill J. Central nervous system germ cell tumors: an update. Curr Opin Oncol. 2014; 26 (6): 622-626.

49. Llewel YN. Manual de diagnóstico clínico. McGraw-Hill: 117-133 
50. Britton $\mathrm{BH}$, Block LD. Vertigo in the pediatric and adolescent age group. Laryngoscope 1988; 98(2): 139146.

51. Gobierno Federal. Guía de diagnóstico, Tratamiento inicial y Prevención de los Tumores Cerebrales Infantiles en el Primer y Segundo Nivel de Atención. Centro Nacional de Excelencia Tecnológica en Salud. México 2008.

52. Chico-Ponce de León F, Castro-Sierra E, PerezpeñaDiazconti M, Gordillo-Domínguez L, Santana-Montero B. and et al. Tumores intracraneanos del niño. Bol MedHosplnfantMex. 2006; 63(6): 367-381.

53. Young P, Tina. MagneticResonancelmaging of Pediatric BrainTumors: State of the Art. Topics in Magnetic Resonance Imaging. 2001; 12(6):411-434. 